\title{
The Risk Averse Investor's Equilibrium Equity Premium in a Semi Martingale Market with Arbitrary Jumps
}

\author{
George M. Mukupa ${ }^{1}$, Elias R. Offen ${ }^{2} \&$ Edward M. Lungu ${ }^{3}$ \\ ${ }^{1}$ Department of Mathematics and Statistics, Mulungushi University, Kabwe-Zambia \\ 2 Department of Mathematics, University of Botswana, Gaborone-Botswana \\ ${ }^{3}$ Department of Mathematics and Applied Mathematics, Botswana International University of Science and Technology, \\ Palapye-Botswana
}

Correspondence: George M. Mukupa, School of Science, Engineering and Technology, Department of Mathematics and Statistics, Mulungushi University, Kabwe-Zambia. E-mail: gmukupa@mu.ac.zm

Received: August 22, 2016 Accepted: November 4, 2016 Online Published: November 29, 2016

doi:10.5539/jmr.v8n6p139 URL: http://dx.doi.org/10.5539/jmr.v8n6p139

\begin{abstract}
In this paper, we study the risk averse investor's equilibrium equity premium in a semi martingale market with arbitrary jumps. We realize that, if we normalize the market, the equilibrium equity premium is consistent to taking the risk free rate $\rho=0$ in martingale markets. We also observe that the value process affects both the diffusive and rare-event premia except for the CARA negative exponential utility function. The bond price always affect the diffusive risk premium for this risk averse investor.
\end{abstract}

Keywords: semi martingale, risk averse, risk premium, jump diffusion

\section{Introduction}

Much work in finance has been based on martingale markets whose future is deemed fair and unpredictable by normalizing prices. This gives investors a fair chance to either gain or lose out on their investments. In our case, we make the market partly predicable in order to give certainty of some degree on a fair compensation an investor receives for having taken up some risk. In this case, we allow $X_{t}$ to be a semimartingale with a decomposition

$$
X_{t}=X_{0}+M+A
$$

such that $M=\left(M_{t}\right)_{0 \leq t \leq T}$ is a square-integrable martingale with $M_{0}=0$ and $A=\left(A_{t}\right)_{0 \leq t \leq T}$ is a predictable process of finite variation $|A|$ with $A_{0}=0$. In this paper, we use the semi martingale approach to determine equilibrium equity premium in a production economy with jumps as opposed to option pricing. The problem of deriving ordering results for option prices has been adressed in several papers [(Karoui \& Shreve, 1998), (Hobson, 1998), (Bellamy, 2000), (Henderson, 2002), (Hendersonn \& Hobson, 2003), (Hendersonnn \& Kluge, 2003), (Moller, 2003), (Eberlein \& Jacod, 1997), (Frey \& Sin, 1999), (Jakubenas, 2002), (Gushchin \& Mordecki, 2002)]. The results for models with nontrivial pricing intervals and the corresponding comparison results are less complete. Comparison results for diffusion processes are discussed in (Karoui \& Shreve, 1998) and nontrivial bounds for stochastic volatility models are given in (Frey \& Sin, 1999). (Bellamy, 2000) (see also (Henderson \& Hobson, 2003)) prove that the price of a European call for a diffusion with jumps is bounded below by the corresponding Black-Scholes price and above by the trivial upper price [see also (Bergman \& Wiener, 1996) and (Hobson, 1998) for alternative proofs]. An important generalization of the technique introduced in (Karoui \& Shreve, 1998) and (Bellamy, 2000) has been established by (Gushchin \& Mordecki, 2002) who derive a general comparison result for one-dimensional semimartingales to some Markov process w.r.t convex ordering of terminal values.

This paper is comparable to (Zhang \& Chang, 2012) and also further elaboration by (Mukupa \& Offen, 2015) and (George $\&$ Offen, 2016) who considered the martingale case of equilibrium equity premium.

\section{Method}

Our price process evolves according to the stochastic differential equation;

$$
d X_{t}=\mu d t+\delta d B_{t}+\left(e^{x}-1\right) d N_{t}-\lambda E\left(e^{x}-1\right) d t .
$$

which is a semi martingale with discontinuities because of the presence of jumps. 
We take $\mu, \delta$ and $\lambda$ as constants and $x$ as a vector of arbitrary distributed jump sizes. The processes $B_{t}$ and $N_{t}$ are independent since Brownian motion is a continuous process while the Poisson process is discrete. The parameter $\lambda$ denotes the frequency of the Poisson process. In this model, we have set the coefficient $\left(e^{x}-1\right)$ in the jump process such that $e^{x}-1=0$ if there is no jump, that is, for $x=0$. E denotes the expectation which makes the process $E\left(e^{x}-1\right)$ deterministic. $d N_{t}$ models the sudden changes as a result of rare events happening and $d B_{t}$ models small continuous changes generated by the noise whose volatility is a constant $\delta$.

Note that the compensated compound Poisson process $\left(e^{x}-1\right) d N_{t}-\lambda E\left(e^{x}-1\right) d t$ has the mean of zero since

$$
E\left[\left(e^{x}-1\right) d N_{t}-\lambda E\left(e^{x}-1\right) d t\right]=E\left(e^{x}-1\right) E\left(d N_{t}\right)-E\left(e^{x}-1\right) E(\lambda d t)=0
$$

and $E\left(d N_{t}\right)=\lambda d t$

To solve

$$
d X_{t}=\mu d t+\delta d B_{t}+\left(e^{x}-1\right) d N_{t}-\lambda E\left(e^{x}-1\right) d t,
$$

we do not need to apply Itô Lemma with Jumps because the diffusion part is a continuous semi martingale whose procedure for solution does not require the integrating factor. We solve for the price process at the terminal time $T$ as follows;

$$
d X_{t}=\left[\mu-\lambda E\left(e^{x}-1\right)\right] d t+\delta d B_{t}+\left(e^{x}-1\right) d N_{t}
$$

Integrating (1), we obtain

$$
X_{T}=X_{t}+\left[\mu-\lambda E\left(e^{x}-1\right)\right] \tau+\delta B_{\tau}+\sum_{i=1}^{N_{\tau}}\left(e^{x_{i}}-1\right),
$$

where $\tau=T-t$ is the investment period.

Suppose that an investor holds two assets, the risk-free asset, $X_{0}(t)$, and the risky asset, $X_{1}(t)=X_{t}$ given by equation (1). The risk-free asset is assumed to evolve according to the equation

$$
d X_{0}(t)=\rho(t) X_{0}(t) d t
$$

where $\rho$ is a constant risk-free rate. Denote $Y_{t}=\left(X_{0}(t), X_{1}(t)\right)$ and the corresponding portfolio by $\phi=(1-\omega, \omega)$ consisting of $1-\omega$ non risky assets and $\omega$ risky assets.

We have, by the self financing strategy, that

$$
d V_{t}=\phi \bullet d Y_{t}
$$

so that the total wealth at any time $t$ is

$$
V_{t}=V_{0}(t)+V_{1}(t)
$$

where $V_{0}(t)$ is the value of the money market account and $V_{1}(t)$ is the value of the investment in the stock market at time $t$. Now

$$
\begin{aligned}
d V_{t} & =d V_{0}(t)+d V_{1}(t) \\
& =(1-\omega) d X_{0}(t)+\omega d X(t) \\
& =(1-\omega)\left(\rho X_{0}(t) d t\right)+\omega\left[\mu-\lambda E\left(e^{x}-1\right)\right] d t+\omega \delta d B_{t}+\omega\left(e^{x}-1\right) d N_{t}
\end{aligned}
$$

Since the equity premium $\hat{\phi}=\mu-\rho$, we have that $\mu=\hat{\phi}+\rho$, hence

$$
d V_{t}=\left[\rho X_{0}(t)-\omega \rho X_{0}(t)+\omega \phi+\omega \rho-\lambda \omega E\left(e^{x}-1\right)\right] d t+\omega \delta d B_{t}+\omega\left(e^{x}-1\right) d N_{t}
$$

The investor's optimal control problem then is to maximize his expected utility function

$$
\max E_{t} \int_{t}^{T} y(t) U\left(r_{t}\right) d t
$$

subject to

$$
d V_{t}=\left[\rho X_{0}(t)-\omega \rho X_{0}(t)+\omega \hat{\phi}+\omega \rho-\lambda \omega E\left(e^{x}-1\right)-r_{t}\right] d t+\omega \delta d B_{t}+\omega\left(e^{x}-1\right) d N_{t}
$$

The wealth ratio $\omega$ and consumption rate $r_{t}$ are control variable. The general equilibrium occur when $\omega=1$. 


\section{Results}

\section{Proposition 1 Equilibrium Equity Premium For CRRA Power Utility Function.}

In a semi martingale market, an investor's equilibrium equity premium with CRRA power utility function $U\left(r_{t}\right)=\frac{r_{t}^{\beta}}{\beta}, 0<$ $\beta<1$, in the production economy with jump diffusion is given by

$$
\hat{\phi}=\rho X_{0}(t)-\rho-(\beta-1) V_{t}^{-1} \delta^{2}+\lambda E\left[\left(e^{x}-1\right)\left(1-V_{t}\left(e^{x}\right)^{\beta-1}\right)\right] .
$$

where $\phi_{\delta}=\rho X_{0}(t)-\rho-(\beta-1) V_{t}^{-1} \delta^{2}$ is the diffusive risk premium and $\phi_{N}=\lambda E\left[\left(e^{x}-1\right)\left(1-V_{t}\left(e^{x}\right)^{\beta-1}\right)\right]$ is the rare-event premium.

Proof. We optimize the investor's utility based on the Hamilton-Jacobi-Bellman (HJB) equation

$$
E_{t}\left[d J+y U\left(r_{t}\right) d t\right]=0 .
$$

Now, Equation (2) can be written as

$$
d V_{t}=d^{*} V_{t}+\omega\left(e^{x}-1\right) d N_{t}
$$

where

$$
d^{*} V_{t}=\left[\rho X_{0}(t)-\omega \rho X_{0}(t)+\omega \phi+\omega \rho-\omega \lambda E\left(e^{x}-1\right)-r_{t}\right] d t+\omega \delta d B_{t}
$$

is the diffusion part. Define

$$
g=J\left(t, d^{*} V_{t}\right)
$$

then Ito's formula gives

$$
d^{*} J=J_{t} d t+J_{V_{t}} d^{*} V_{t}+\frac{1}{2} J_{V_{t} V_{t}}\left(d^{*} V_{t}\right)^{2} .
$$

The generalized process of equation (3) gives

$$
\begin{aligned}
d J & =d^{*} J+\left[J\left(V_{t}\left(1+\omega\left(e^{x}-1\right)\right), t\right)-J\left(V_{t}, t\right)\right] d N_{t} \\
& =J_{t} d t+J_{V_{t}} d^{*} V_{t}+\frac{1}{2} J_{V_{t} V_{t}}\left(d^{*} V_{t}\right)^{2}+\left[J\left(V_{t}\left(1+\omega\left(e^{x}-1\right)\right), t\right)-J\left(V_{t}, t\right)\right] d N_{t} \\
& =J_{t} d t+J_{V_{t}}\left[\left[\rho X_{0}(t)-\omega \rho X_{0}(t)+\omega \phi+\omega \rho-\omega \lambda E\left(e^{x}-1\right)-r_{t}\right] d t+\omega \delta d B_{t}\right]+\frac{1}{2} J_{V_{t} V_{t}}\left[\omega^{2} \delta^{2} d t\right]+\left[J\left(V_{t}\left(1+\omega\left(e^{x}-1\right)\right), t\right)-J\left(V_{t}, t\right)\right] d N_{t} \\
& =J_{t} d t+J_{V_{t}}\left[\rho X_{0}(t)-\omega \rho X_{0}(t)+\omega \phi+\omega \rho-\omega \lambda E\left(e^{x}-1\right)-r_{t}\right] d t+J_{V_{t}} \omega \delta d B_{t}+\frac{1}{2} J_{V_{t} V_{t}}\left[\omega^{2} \delta^{2} d t\right]+\left[J\left(V_{t}\left(1+\omega\left(e^{x}-1\right)\right), t\right)-J\left(V_{t}, t\right)\right] d N_{t} .
\end{aligned}
$$

Equation (2) can now be written as

$$
\begin{aligned}
& J_{t} d t+J_{V_{t}}\left[\rho X_{0}(t)-\omega \rho X_{0}(t)+\omega \phi+\omega \rho-\omega \lambda E\left(e^{x}-1\right)-r_{t}\right] d t+\frac{1}{2} J_{V_{t} V_{t}} \omega^{2} \delta^{2} d t \\
& +E\left[J\left(V_{t}\left(1+\omega\left(e^{x}-1\right)\right), t\right)-J\left(V_{t}, t\right)\right] \lambda d t+y U\left(r_{t}\right) d t=0 \\
& J_{t}+J_{V_{t}}\left[\rho X_{0}(t)-\omega \rho X_{0}(t)+\omega \phi+\omega \rho-\omega \lambda E\left(e^{x}-1\right)-r_{t}\right]+\frac{1}{2} J_{V_{t} V_{t}} \omega^{2} \delta^{2} \\
& +\lambda E\left[J\left(V_{t}\left(1+\omega\left(e^{x}-1\right)\right), t\right)-J\left(V_{t}, t\right)\right]+y U\left(r_{t}\right)=0
\end{aligned}
$$

To find the optimal values, we solve

$$
\begin{gathered}
\max _{\left(r_{t}, \omega\right)}\left\{J_{t}+J_{V_{t}}\left[\rho X_{0}(t)-\omega \rho X_{0}(t)+\omega \phi+\omega \rho-\omega \lambda E\left(e^{x}-1\right)-r_{t}\right]+\frac{1}{2} J_{V_{t} V_{t}} \omega^{2} \delta^{2}\right. \\
\left.+\lambda E\left[J\left(V_{t}\left(1+\omega\left(e^{x}-1\right)\right), t\right)-J\left(V_{t}, t\right)\right]+y U\left(r_{t}\right)=0\right\},
\end{gathered}
$$

by taking partial derivatives with respect to $r_{t}$ and $\omega$ to obtain the first order conditions

$$
\begin{aligned}
& -J_{V_{t}}+y U\left(r_{t}\right)=0 \\
& \left.-\rho X_{0}(t)+\phi+\rho-\lambda E\left(e^{x}-1\right)\right] J_{V_{t}}+J_{V_{t} V_{t}} \omega \delta^{2}+\lambda E\left[J_{V_{t}}\left(V_{t}\left(1+\omega\left(e^{x}-1\right)\right), t\right) V_{t}\left(e^{x}-1\right)\right]=0
\end{aligned}
$$


Solving for $\hat{\phi}$ from the second equation and taking the equilibrium condition $\omega=1$ yields the general equilibrium equity premium

$$
\hat{\phi}=\rho X_{0}(t)-\rho+\lambda E\left(e^{x}-1\right)-\frac{J_{V_{t} V_{t}}}{J_{V_{t}}} \delta^{2}-\frac{\lambda}{J_{V_{t}}} E\left[J_{V_{t}}\left(V_{t} e^{x}, t\right) V_{t}\left(e^{x}-1\right)\right] .
$$

Substituting this $\hat{\phi}$ into the Bellman equation

$$
\begin{aligned}
J_{t}+J_{V_{t}} \rho X_{0}(t) & -J_{V_{t}} \rho X_{0}(t)+J_{V_{t}}\left[\rho X_{0}(t)-\rho+\lambda E\left(e^{x}-1\right)-\frac{J_{V_{V} V_{t}}}{J_{V_{t}}} \delta^{2}-\frac{\lambda}{J_{V_{t}}} E\left[J_{V_{t}}\left(V_{t} e^{x}, t\right) V_{t}\left(e^{x}-1\right)\right]\right. \\
& \left.+J_{V_{t}} \rho-J_{V_{t}} \lambda E\left(e^{x}-1\right)-J_{V_{t}} r_{t}+\frac{1}{2} J_{V_{t} V_{t}} \delta^{2}+\lambda E\left[J\left(V_{t} e^{x}\right), t\right)-J\left(V_{t}, t\right)\right]+y U\left(r_{t}\right)=0
\end{aligned}
$$

which simplifies to the integro p.d.e

$$
\begin{aligned}
J_{t}+J_{V_{t}} \rho X_{0}(t) & \left.-J_{V_{t}} r_{t}-\frac{1}{2} J_{V_{t} V_{t}} \delta^{2}-\lambda E\left[J_{V_{t}}\left(V_{t} e^{x}, t\right) V_{t}\left(e^{x}-1\right)\right]+\lambda E\left[J\left(V_{t} e^{x}\right), t\right)-J\left(V_{t}, t\right)\right] \\
& +y U\left(r_{t}\right)=0
\end{aligned}
$$

Consider now the power utility function

$$
U\left(r_{t}\right)=\frac{r_{t}^{\beta}}{\beta}, \quad 0<\beta<1,
$$

we solve for $J\left(V_{t}, t\right)$ based on the indirect utility

$$
J\left(V_{t}, t\right)=Q(t) \frac{V_{t}^{\beta}}{\beta}
$$

The optimal consumption will be solved from the first order condition (1) as:

$$
y U^{\prime}\left(r_{t}\right)=J_{V_{t}}
$$

which implies

$$
\begin{aligned}
y\left[r_{t}^{\beta-1}\right] & =Q(t) V_{t}^{\beta-1} \\
r_{t} & =\left(\frac{Q(t) V_{t}^{\beta-1}}{y}\right)^{\frac{1}{\beta-1}}
\end{aligned}
$$

and therefore

$$
r_{t}=\left(\frac{Q(t)}{y}\right)^{\frac{1}{\beta-1}} V_{t}
$$

is the optimal consumption we require.

Substituting the functions $J\left(V_{t}, t\right)=Q(t) \frac{V_{t}^{\beta}}{\beta}, J_{V_{t}}=Q(t) V_{t}^{\beta-1}$ and $J_{V_{t} V_{t}}=(\beta-1) Q(t) V_{t}^{\beta-2}$ into the integro p.d.e gives

$$
\begin{aligned}
\frac{Q(t) V_{t}^{\beta}}{\beta}+\rho X_{0}(t) Q(t) V_{t}^{\beta-1} & -\left(\frac{Q(t)}{y}\right)^{\frac{1}{\beta-1}} V_{t} Q_{t} V_{t}^{\beta-1}-\frac{1}{2} \delta^{2}(\beta-1) Q(t) V_{t}^{\beta-2}-\lambda E\left[Q(t)\left(V_{t} e^{x}\right)^{\beta-1} V_{t}\left(e^{x}-1\right)\right] \\
& +\lambda E\left[\frac{Q(t) V_{t}^{\beta}\left(e^{\beta x}-1\right)}{\beta}\right]+\frac{y}{\beta}\left[\left(\frac{Q(t)}{y}\right)^{\frac{\beta}{\beta-1}} V_{t}^{\beta}\right]=0
\end{aligned}
$$

differentiating with respect to $V_{t}$ and dividing through by $V_{t}^{\beta-1}$ gives the terminal conditions

$$
\begin{aligned}
Q(t)+a Q(t)+y\left[\left(\frac{Q(t)}{y}\right)^{\frac{\beta}{\beta-1}}\right] & =0 . \\
Q(T) & =0
\end{aligned}
$$


where

$$
\begin{aligned}
a & =\rho X_{0}(t)(\beta-1) V_{t}^{-1}-\beta\left(\frac{Q(t)}{y}\right)^{\frac{1}{\beta-1}}-\frac{1}{2} \delta^{2}(\beta-1)(\beta-2) V_{t}^{-2}-\lambda \beta E\left[e^{x(\beta-1)}\left(e^{x}-1\right)\right] \\
& +\lambda E\left[e^{x \beta}-1\right]
\end{aligned}
$$

Substituting $J\left(V_{t}, t\right)=Q(t) \frac{V_{t}^{\beta}}{\beta}, J_{V_{t}}=Q(t) V_{t}^{\beta-1}$ and $J_{V_{t} V_{t}}=(\beta-1) Q(t) V_{t}^{\beta-2}$ into the general equilibrium equity premium gives us the equity premium for the power utility function as;

$$
\phi=\rho X_{0}(t)-\rho-(\beta-1) V_{t}^{-1} \delta^{2}+\lambda E\left[\left(e^{x}-1\right)\left(1-V_{t}\left(e^{x}\right)^{\beta-1}\right)\right] .
$$

Notice here that, if we normalize the market by $X_{0}(t)=1$, the equilibrium equity premium will be given by

$$
\phi=-(\beta-1) V_{t}^{-1} \delta^{2}+\lambda E\left[\left(e^{x}-1\right)\left(1-V_{t}\left(e^{x}\right)^{\beta-1}\right)\right] .
$$

This is consistent to taking $\rho=0$ as in martingale markets. We also observe that the value process affects both the diffusive and rare-event premia. If the value process $V_{t}=0$, the investor's equilibrium equity premium becomes undefined. We therefore urge investors under this utility function to increase on the value process as this reduces the diffusive risk significantly.

\section{Proposition 2 Equilibrium Equity Premium For Negative Exponential Utility Function}

Under the CARA negative exponential utility function $U\left(r_{t}\right)=-e^{-\alpha r_{t}}, \alpha>0$, the investor's equilibrium equity premium in the production economy with jump diffusion is given by

$$
\phi=\rho X_{0}(t)-\rho+\alpha \delta^{2}+\lambda E\left[\left(e^{x}-1\right)\left(1-\frac{V_{t} e^{-\alpha V_{t} e^{x}}}{e^{-\alpha V_{t}}}\right)\right]
$$

Proof. Suppose that the investor's utility function is given by

$$
U\left(r_{t}\right)=-e^{-\alpha r_{t}}, \alpha>0,
$$

which is the exponential utility function. We observe that,

$$
\begin{gathered}
U^{\prime}\left(r_{t}\right)=\alpha e^{-\alpha r_{t}}>0, \\
U^{\prime \prime}\left(r_{t}\right)=-\alpha^{2} e^{-\alpha r_{t}}<0,
\end{gathered}
$$

which implies the utility function is concave.

We solve for $J\left(V_{t}, t\right)$ by conjecturing that

$$
J\left(V_{t}, t\right)=-Q_{t} e^{-\alpha V_{t}},
$$

and use the first order condition (1) as follows

$$
y U^{\prime}\left(r_{t}\right)=J_{V_{t}}
$$

which implies

$$
y\left(\alpha e^{-\alpha r_{t}}\right)=\alpha Q_{t} e^{-\alpha V_{t}} .
$$

Solving for $r_{t}$ the optimal consumption for this investor is given by:

$$
r_{t}=\frac{-1}{\alpha} \ln \left[\frac{Q_{t} e^{-\alpha V_{t}}}{y}\right], \quad \alpha>0 .
$$

Substituting the functions $J\left(V_{t}, t\right)=-Q_{t} e^{-\alpha V_{t}}, J_{V_{t}}\left(V_{t}, t\right)=\alpha Q_{t} e^{-\alpha V_{t}}$ and $J_{V_{t} V_{t}}\left(V_{t}, t\right)=-\alpha^{2} Q_{t} e^{-\alpha V_{t}}$ into the integro-partial differential equation gives

$$
\begin{aligned}
-Q_{t} e^{-\alpha V_{t}}+\rho X_{0}(t) \alpha Q_{t} e^{-\alpha V_{t}} & +Q_{t} e^{-\alpha V_{t}} \ln \left[\frac{Q_{t} e^{-\alpha V_{t}}}{y}\right]+\frac{1}{2} \alpha^{2} Q_{t} e^{-\alpha V_{t}} \delta^{2}-\lambda E\left[\alpha Q_{t} e^{-\alpha V_{t} e^{x}} V_{t}\left(e^{x}-1\right)\right] \\
& +\lambda E\left[\alpha Q_{t} e^{-\alpha V_{t} e^{x}}+Q_{t} e^{-\alpha V_{t}}\right]-y e^{\ln \left[\frac{Q_{t} e^{-\alpha V_{t}}}{y}\right]}=0 .
\end{aligned}
$$


Differentiating with respect to $V_{t}$ and dividing through by $\alpha e^{-\alpha V_{t}}$ gives the terminal conditions

$$
Q_{t}+a Q_{t}-\frac{y e^{-\alpha}}{\alpha e^{-\alpha V_{t}}}=0 \quad \text { and } \quad Q_{T}=0
$$

where

$$
\begin{aligned}
a & =-\alpha \rho X_{0}(t)-\ln \left[\frac{Q_{t} e^{-\alpha V_{t}}}{y}\right]-\frac{1}{2} \alpha^{2} \delta^{2}-\frac{\lambda}{e^{-\alpha V_{t}}} E\left[e^{-\alpha V_{t} e^{x}}\left(e^{x}-1\right)\left(1-V_{t} \alpha e^{x}\right)\right] \\
& +\frac{\lambda}{e^{-\alpha V_{t}}} E\left[-\alpha e^{x} e^{-\alpha V_{t} e^{x}}-e^{-\alpha V_{t}}\right]
\end{aligned}
$$

Substituting $J\left(V_{t}, t\right)=-Q_{t} e^{-\alpha V_{t}}, J_{V_{t}}\left(V_{t}, t\right)=\alpha Q_{t} e^{-\alpha V_{t}}$ and $J_{V_{t} V_{t}}\left(V_{t}, t\right)=-\alpha^{2} Q_{t} e^{-\alpha V_{t}}$ into the general equilibrium equity premium gives

$$
\phi=\rho X_{0}(t)-\rho+\alpha \delta^{2}+\lambda E\left[\left(e^{x}-1\right)\left(1-\frac{V_{t} e^{-\alpha V_{t} e^{x}}}{e^{-\alpha V_{t}}}\right)\right]
$$

The wealth process affects only the rare event premium. We notice here that, if the wealth process $V_{t}=0$, the rare event premium is also zero. The diffusive risk for this utility is always positive regardless of how volatile the process becomes. In fact, the more the process becomes volatile, the more the investor is exposed to the diffusive risk.

Proposition 3 Equilibrium Equity Premium For Square Root Utility Function.

In the production economy with jump diffusion, the investor's equilibrium equity premium with square root utility function $U\left(r_{t}\right)=\sqrt{r_{t}}, r_{t}>0$, is given by

$$
\phi=\rho X_{0}(t)-\rho+\frac{\delta^{2}}{2 V_{t}}+\lambda E\left[\left(e^{x}-1\right)\left(1-V_{t} e^{-\frac{1}{2} x}\right)\right]
$$

where $\phi_{\delta}=\rho X_{0}(t)-\rho+\frac{\delta^{2}}{2 V_{t}}$ is the diffusive risk premium and $\phi_{N}=\lambda E\left[\left(e^{x}-1\right)\left(1-V_{t} e^{-\frac{1}{2} x}\right)\right]$ is the rare-event premium. Proof. For the square root utility function

$$
U\left(r_{t}\right)=\sqrt{r_{t}}, r_{t}>0
$$

the optimal consumption

$$
r_{t}=\left(\frac{y \sqrt{V_{t}}}{Q_{t}}\right)^{2}=\frac{y^{2} V_{t}}{Q_{t}^{2}}
$$

which we have found by taking $J\left(V_{t}, t\right)$ of the form

$$
J\left(V_{t}, t\right)=Q_{t} V_{t}^{\frac{1}{2}}
$$

We note that in this case

$$
U^{\prime}\left(r_{t}\right)=\frac{1}{2}\left(r_{t}\right)^{-\frac{1}{2}}=\frac{1}{2 \sqrt{r_{t}}}>0
$$

and

$$
U^{\prime \prime}\left(r_{t}\right)=-\frac{1}{4}\left(r_{t}\right)^{-\frac{1}{2}-1}=-\frac{1}{4}\left(r_{t}\right)^{-\frac{3}{2}}=-\frac{1}{4} \times \frac{1}{\sqrt{r_{t}^{3}}}=-\frac{1}{4 \sqrt{r_{t}^{3}}}<0
$$

implying that this utility is a concave function. The coefficient of aversion is

$$
\begin{aligned}
R R A & =-\frac{U^{\prime \prime} r_{t}}{U^{\prime}} \\
& =\frac{\frac{r_{t}}{4 \sqrt{r_{t}^{3}}}}{\frac{1}{2 \sqrt{r_{t}}}}=\frac{r_{t}}{4 \sqrt{r_{t}^{3}}} \times 2 \sqrt{r_{t}} \\
& =\frac{1}{2}>0 .
\end{aligned}
$$


It is easy to see that the RRA is $\frac{1}{2}$ by virtue of square root since the CRRA family is of form $U(c)=c^{\beta}$ for some $R R A=\beta>0$.

Substituting $J_{t}, J_{V_{t}}, J_{V_{t} V_{t}}$ and $r_{t}$ into the integro-partial differential equation

$$
\begin{aligned}
J_{t}+\rho V_{t} J_{V_{t}}-\frac{1}{2} \delta^{2} V_{t}^{2} J_{V_{t} V_{t}} & -\lambda V_{t} E\left[J_{V_{t}}\left(V_{t} e^{x}, t\right)\left(e^{x}-1\right)\right]+\lambda E\left[J\left(V_{t} e^{x}, t\right)\right] \\
& -\lambda J_{t}-J_{V_{t}} r_{t}+y U\left(r_{t}\right)=0 .
\end{aligned}
$$

This gives

$$
\begin{aligned}
Q_{t} V_{t}^{\frac{1}{2}}+\rho X_{0}(t) \frac{Q_{t}}{2 V_{t}^{\frac{1}{2}}} & -\frac{y^{2} V_{t}^{\frac{1}{2}}}{2 Q_{t}}+\frac{\delta^{2} Q_{t}}{8 V_{t}^{\frac{3}{2}}}-\lambda V_{t}^{\frac{1}{2}} E\left[\frac{Q_{t}}{2\left(e^{x}\right)^{\frac{1}{2}}}\left(e^{x}-1\right)\right] \\
& +\lambda E\left[Q_{t} V_{t}^{\frac{1}{2}}\left(e^{\frac{1}{2} x}-1\right)\right]+\frac{y^{2} V_{t}^{\frac{1}{2}}}{Q_{t}}=0
\end{aligned}
$$

Differentiating with respect to $V_{t}$ and dividing through by $V_{t}^{-\frac{1}{2}}$ yields

$$
\frac{1}{2} Q_{t}-\frac{1}{4} \rho X_{0}(t) Q_{t} V_{t}^{-1}-\frac{y^{2}}{4 Q_{t}}-\frac{3}{16} \delta^{2} Q_{t} V_{t}^{-2}-\frac{1}{4} \lambda Q_{t} E\left[\frac{e^{x}-1}{e^{\frac{1}{2} x}}\right]+\frac{1}{2} \lambda E\left[Q_{t}\left(e^{\frac{1}{2} x}-1\right)\right]+\frac{y^{2}}{2 Q_{t}}=0 .
$$

and thus

$$
\begin{aligned}
\frac{1}{2} Q_{t}+a Q_{t}+\frac{y^{2}}{2 Q_{t}} & =0 \\
Q(T) & =0
\end{aligned}
$$

where

$$
a=-\frac{1}{4} \rho X_{0}(t) V_{t}^{-1}-\frac{y^{2}}{4 Q_{t}^{2}}-\frac{3}{16} \delta^{2} V_{t}^{-2}-\frac{1}{4} \lambda E\left[\frac{e^{x}-1}{e^{\frac{1}{2} x}}\right]+\frac{1}{2} \lambda E\left[e^{\frac{1}{2} x}-1\right]
$$

are terminal conditions.

Substituting into the general equilibrium equity premium formula gives the equilibrium equity premium for the square root utility function as

$$
\phi=\rho X_{0}(t)-\rho+\frac{\delta^{2}}{2 V_{t}}+\lambda E\left[\left(e^{x}-1\right)\left(1-V_{t} e^{-\frac{1}{2} x}\right)\right]
$$

For this utility function, the value process affects both the diffusive and rare event premia. Again, if the wealth process $V_{t}=0$, the equilibrium equity premium is undefined. If the wealth process increases, the diffusive risk reduces and vice-versa. The rare event premium also reduces with the increase in the wealth value.

Proposition 4 Equilibrium Equity Premium For Quadratic Utility Function.

An investor's equilibrium equity premium with quadratic utility function $U\left(r_{t}\right)=r_{t}-a r_{t}^{2}, a>0$ in the production economy with jump diffusion is given by

$$
\phi=\rho X_{0}(t)-\rho+\frac{2 a \delta^{2}}{1-2 a V_{t}}+\lambda E\left[\left(e^{x}-1\right)\left(1-\frac{V_{t}\left(1-2 a V_{t} e^{x}\right)}{1-2 a V_{t}}\right)\right]
$$

where $\phi_{\delta}=\rho X_{0}(t)-\rho+\frac{2 a \delta^{2}}{1-2 a V_{t}}$ is the diffusive risk premium and $\phi_{N}=\lambda E\left[\left(e^{x}-1\right)\left(1-\frac{V_{t}\left(1-2 a V_{t} e^{x}\right)}{1-2 a V_{t}}\right)\right]$ is the rare-event premium.

Proof. Suppose now that this investor consumed quadratically from the investment, that is

$$
U\left(r_{t}\right)=r_{t}-a r_{t}^{2}, a>0,
$$


then

$$
\begin{aligned}
& U^{\prime}\left(r_{t}\right)>0, \\
& U^{\prime \prime}\left(r_{t}\right)<0
\end{aligned}
$$

implying $U\left(r_{t}\right)$ is a concave utility function by virtue of $U^{\prime \prime}\left(r_{t}\right)$ being negative.

We solve for $J\left(V_{t}, t\right)$ by conjecturing that

$$
\begin{gathered}
J\left(V_{t}, t\right)=Q_{t}\left(V_{t}-a V_{t}^{2}\right) \\
=Q_{t} V_{t}-a Q_{t} V_{t}^{2}
\end{gathered}
$$

so that

$$
J_{V_{t}}\left(V_{t}, t\right)=Q_{t}-2 a Q_{t} V_{t}
$$

and

$$
J_{V_{t} V_{t}}\left(V_{t}, t\right)=-2 a Q_{t}
$$

From the first order conditions, the optimal consumption result as

$$
r_{t}=\frac{y-Q_{t}+2 a Q_{t} V_{t}}{2 a y}
$$

which is affected by both the time preference function $y(t)$ and $V_{t}$ the total wealth at any time $t$.

We substitute $J_{t}, J_{V_{t}}$ and $J_{V_{t} V_{t}}$ into the integro-partial differencial equation to obtain

$$
\begin{gathered}
Q_{t} V_{t}-a Q_{t} V_{t}^{2}+\rho X_{0}(t) Q_{t}-2 a \rho X_{0}(t) Q_{t} V_{t}-\frac{1}{2 a y}\left[\left(y-Q_{t}+2 a Q_{t} V_{t}\right)\left(Q_{t}-2 a Q_{t} V_{t}\right)\right] \\
+a \delta^{2} Q_{t}-\lambda E\left[\left(Q_{t}-2 a Q_{t} V_{t} e^{x}\right) V_{t}\left(e^{x}-1\right)\right]+\lambda E\left[Q_{t} V_{t}\left(e^{x}-1\right)-a Q_{t} V_{t}^{2}\left(e^{2 x}-1\right)\right] \\
+\frac{\left(y-Q_{t}+2 a Q_{t} V_{t}\right)\left(y+Q_{t}-2 a Q_{t} V_{t}\right)}{4 a y}=0 .
\end{gathered}
$$

Differentiating with respect to $V_{t}$, we have the terminal conditions

$$
\begin{gathered}
Q_{t}-2 a Q_{t} V_{t}-2 a \rho X_{0}(t) Q_{t}-\frac{1}{2 a y}\left[\left(y-Q_{t}+2 a Q_{t} V_{t}\right)\left(-2 a Q_{t}\right)+\left(Q_{t}-2 a Q_{t} V_{t}\right)\left(2 a Q_{t}\right)\right] \\
-\lambda E\left[\left(Q_{t}-2 a Q_{t} V_{t} e^{x}\right)\left(e^{x}-1\right)+V_{t}\left(e^{x}-1\right)\left(-2 a Q_{t} e^{x}\right)\right]+\lambda E\left[Q_{t}\left(e^{x}-1\right)-2 a Q_{t} V_{t}\left(e^{2 x}-1\right)\right] \\
+\frac{\left(y-Q_{t}+2 a Q_{t} V_{t}\right)\left(-2 a Q_{t}\right)+\left(y+Q_{t}-2 a Q_{t} V_{t}\right)\left(2 a Q_{t}\right)}{4 a y}=0
\end{gathered}
$$

and

$$
Q(T)=0
$$

Substituting into the general equity premium gives us the desired result

$$
\phi=\rho X_{0}(t)-\rho+\frac{2 a \delta^{2}}{1-2 a V_{t}}+\lambda E\left[\left(e^{x}-1\right)\left(1-\frac{V_{t}\left(1-2 a V_{t} e^{x}\right)}{1-2 a V_{t}}\right)\right]
$$

The equity premium is always positive regardless of the wealth value. Even when the wealth process becomes zero, the diffusive and rare events premia are never zero.

\section{Conclusions}

In the semi martingale market, the equilibrium equity premium for risk averse investors is affected by the wealth value except for the CRRA negative exponential utility function. For the power and square root utility, we realised that, if the value process $V_{t}=0$, the investor's equilibrium equity premium becomes undefined. If the wealth process increases, the risks reduces and vice-versa.The quadratic utility is the only utility affecting the premium uniquely. The risks for this premium are always positive regardless of the wealth process. 


\section{Acknowledgements}

We wish to thank the Editor and the reviewers for their comments.

\section{References}

Bellamy, N. (2000). Incompleteness of markets driven by a mixed diffusion. Finance Stoch., 4(2), 209-222.

Bergman, Y. Z., \& Weiner, Z. (1996). General properties of option prices. J. Finance, 51, 1573-1610.

Eberlein, E., \& Jacod, J. (1997).On the range of options prices. Finance Stoch., I(2), 131-140.

Karoui, N., \& Shreve, S. E. (1998). Robustness of the Black \& Scholes formula. Math. Finance, 8(2), 93-126.

Frey, R., \& Sin, C. A. (1999). Bounds on european option prices under stochastic volatility. Math. Finance, 9(2), 97-116.

George, M. M., \& Offen, E. R. (2016). Equity premium under normally distributed jump sizes in a production economy with jumps. International Journal of Applied Mathematics and Statistics (IJAMAS), 54(2), 27-41.

Gushchin, A. A., \& Mordecki, E. (2002). Bounds on option prices for semi martingale market models. Proc. Steklov Inst. Math., 237, 73-113

Henderson, V. (2002). Analytical comparisons of option prices in stochastic volatility models, Oxford Financial Research Centre Preprint 2002-MF-03.

Henderson, V., \& Kluge, T. (2003). A comparison of q-optimal option prices in a stochastic volatility model with correlation. Oxford Financial Research Centre Preprint 2003-MF-02.

Henderson, V., \& Hobson, D. G. (2003). Coupling and option price comparisons in a jump diffusion model. Stochastics Rep., 75(3), 79-101

Hobson, D. G. (1998). Volatility misspecification, option pricing and superreplication via coupling. Ann. Appl. Probab., 8(1), 193-205.

Jakubenas, P. (2002). On option pricing in certain incomplete markets. proc. steklov inst., Steklov Inst. Math., 237, 114-133.

Moller, T. (2003). Stochastic orders in dynamic reinsurance markets. preprint.

Mukupa, G., \& Offen, E. (2015). The impact of utility functions on the equilibrium equity premium in a production economy with jump diffusion. IAENG International Journal of Applied Mathematics, 45(2), 120-127.

Zhang, J. E., Zhao, H. M., \& Chang, E. C. (2012). Equilibrium asset and option pricing under jump diffusion. The University of Hong Kong, Mathematical Finance, 22,(3), 538-568.

\section{Copyrights}

Copyright for this article is retained by the author(s), with first publication rights granted to the journal.

This is an open-access article distributed under the terms and conditions of the Creative Commons Attribution license (http://creativecommons.org/licenses/by/4.0/). 